Bull. Austral. Math. Soc.

20D15, 20B05

VOL. $73(2005)$ [219-230]

\title{
ON THE NUMBER OF CONJUGACY CLASSES OF NORMALISERS IN A FINITE $p$-GROUP
}

\author{
Norberto Gavioli, Leire Legarreta, Carmela Sica and Maria Tota
}

\begin{abstract}
In 1996 Poland and Rhemtulla proved that the number $\nu(G)$ of conjugacy classes of non-normal subgroups of a non-Hamiltonian nilpotent group $G$ is at least $c-1$, where $c$ is the nilpotency class of $G$. In this paper we consider the map that associates to every conjugacy class of subgroups of a finite p-group the conjugacy class of the normaliser of any of its representatives. In spite of the fact that this map need not be injective, we prove that, for $p$ odd, the number of conjugacy classes of normalisers in a finite p-group is at least $c$ (taking into account the normaliser of the normal subgroups). In the case of $p$-groups of maximal class we can find a better lower bound that depends also on the prime $p$.
\end{abstract}

\section{INTRODUCTION}

For any group $G$, we denote respectively by $\nu(G)$ and $\omega(G)$ the number of conjugacy classes of non-normal subgroups of $G$ and the number of conjugacy classes of subgroups of $G$ that are the normalisers of some subgroup of $G$.

The inequality $\nu(G) \geqslant c-1$ has been established to hold by Poland and Rhemtulla in [7] when $G$ is a nilpotent group of class $c$ that is not Hamiltonian. More recently Fernández-Alcober and Legarreta [4] found a sharper bound for finite p-groups: $\nu(G)$ $\geqslant p(k-1)+1$, where $k$ is defined by $\left|G^{\prime}\right|=p^{k}$ and $p$ is an odd prime.

Clearly, for any conjugacy class $K$ of subgroups of $G$, there exists one conjugacy class $\rho(K)$ of the normaliser of a representative of $K$. In spite of the fact that the function $\rho$ is not injective, there exists computational evidence, with very few exceptions, that for a finite $p$-group, with $p$ odd, the number $\omega(G)$ satisfies the similar inequality $\omega(G) \geqslant p(k-1)+2$ (note that the group $G$ is the normaliser of the normal subgroups).

Received 17th October, 2005

The first author is member of INdAM-GNSAGA. Research supported by the Spanish Ministry of Science and Education, grant MTM2004-04665, partly with FEDER funds, and by the University of the Basque Country, grant 1/UPV 00127.310-E-15280/2003 and by the Italian Ministry of Education, University and Research.

The authors would like to thank Professor Gustavo Fernández-Alcober for the helpful suggestions regarding p-groups of maximal class and related references.

Copyright Clearance Centre, Inc. Serial-fee code: 0004-9727/05 \$A2.00+0.00. 
In the present paper we find lower bounds for $\omega(G)$, when $G$ is a finite $p$-group and $p$ is an odd prime, depending on the structure of $G$. These bounds are surprisingly similar to those found for $\nu(G)$.

In the first section we prove that the mentioned inequality $\omega(G) \geqslant p(k-1)+2$ holds for any $p$-group $G$ of maximal class not isomorphic to $Q_{2^{n}}$ (see Theorem 4 ) and we give an example where the inequality becomes an equality.

In the second section we study the general case and we show that there exists a bound similar to the one given by Poland and Rhemtulla: in Theorem 6 we show that when $p$ is an odd prime, the inequality $\omega(G) \geqslant c$ holds for all finite p-groups having nilpotency class $c$. The $p$-group of order $p^{3}$ and exponent $p^{2}$ is an example where equality holds.

We acknowledge the use of the library of groups of small order, that is part of computer package GAP [5], as a useful tool to find two groups of order $3^{6}$ that do not satisfy the inequality $\omega(G) \geqslant p(k-1)+2$.

\section{1. $p$-GROUPS OF MAXIMAL CLASS}

In the present section $G$ will denote a finite $p$-group of maximal class of order $p^{n}$ and $n \geqslant 4$.

We start by enumerating some well known results for $p$-groups of maximal class mainly due to Blackburn [2]. For the proofs of the statements the reader is referred to $[3]$.

Notation. Let us denote $G_{i}=\gamma_{i}(G)$ for any $2 \leqslant i \leqslant n, G_{0}=G$ and $G_{1}=C_{G}\left(G_{2} / G_{4}\right)$, which means that $G_{1}$ consists of the elements $x \in G$ such that $\left[x, G_{2}\right] \leqslant G_{4}$. Furthermore, in the same way as the subgroup $G_{1}$ is defined, we consider more generally the so-called two-step centralisers $C_{G}\left(G_{i} / G_{i+2}\right)$ for $1 \leqslant i \leqslant n-2$.

DEFINITION 1: We say $s \in G$ is a uniform element if $s \notin \bigcup_{i=2}^{n-2} C_{G}\left(G_{i} / G_{i+2}\right)$.

THEOREM 1. Let $G$ be a $p$-group of maximal class of order $p^{n}$ and suppose that $s$ is a uniform element in $G$. The following properties hold:

(i) $C_{G}(s)=\langle s\rangle Z(G)$.

(ii) $s^{p} \in Z(G)$ and consequently $o(s) \leqslant p^{2}$ and $\left|C_{G}(s)\right|=p^{2}$.

(iii) The conjugates of $s$ are exactly the elements in the coset $s G_{2}$.

(iv) If $1 \leqslant i \leqslant n-2$ and $g \in G_{i} \backslash G_{i+1}$ then $[s, g] \in G_{i+1} \backslash G_{i+2}$.

DEFINITION 2: We define the degree of commutativity of $G$, which we denote by $l(G)$ as follows,

$$
l(G)=\max \left\{k \leqslant n-2 \mid\left[G_{i}, G_{j}\right] \leqslant G_{i+j+k} \text { for all } i, j \geqslant 1\right\} .
$$


It is clear that $l(G)=n-2$ if and only if $G_{1}$ is Abelian. Since the terms of the lower central series of any group $G$ satisfy the condition $\left[\gamma_{i}(G), \gamma_{j}(G)\right] \leqslant \gamma_{i+j}(G)$ for all $i, j \geqslant 1$, it is clear that for any $p$-group of maximal class, $l(G) \geqslant 0$.

Definition 3: A p-group of maximal class is called exceptional if $l(G)=0$.

THEOREM 2. Let $G$ be a p-group of maximal class. The following statements hold:

(i) $G$ has uniform elements.

(ii) $l(G / Z(G)) \geqslant 1$.

(iii) If $|G| \leqslant p^{p+1}$ then $\exp (G / Z(G))=\exp G_{2}=p$, in particular, $\exp G_{n-2}=p$.

(iv) If $|G| \geqslant p^{p+2}$ then $l(G) \geqslant 1$. Furthermore, $\exp G_{n-p+1}=p$ and in particular, if $p>2, \exp G_{n-2}=p$.

Lemma 1. Let $G$ be a $p$-group. If $g$ is an element in $G$ of order $p$ then $N_{G}(\langle g\rangle)$ $=C_{G}(g)$.

Proof: $\left(N_{G}(\langle g\rangle)\right) /\left(C_{G}(g)\right) \lesssim \operatorname{Aut}(\langle g\rangle) \cong$ Aut $C_{p} \cong \mathbb{U}(\mathbb{Z} / p \mathbb{Z})$, where $|\mathbb{U}(\mathbb{Z} / p \mathbb{Z})|$ $=p-1$ and $\left|\left(N_{G}(\langle g\rangle)\right) /\left(C_{G}(g)\right)\right|$ is a power of $p$. So, $\left|\left(N_{G}(\langle g\rangle)\right) /\left(C_{G}(g)\right)\right|=1$ and, in consequence, $N_{G}(\langle g\rangle)=C_{G}(g)$.

Lemma 2. Let $G$ be a p-group of maximal class. Then:

(i) The only normal subgroups of $G$ are the $\gamma_{i}(G)$ and the maximal subgroups of $G$. More precisely, if $N$ is a normal subgroup of $G$ of index $p^{i} \geqslant p^{2}$ then $N=\gamma_{i}(G)$.

(ii) If $N$ is a normal subgroup of $G$ of index $\geqslant p^{2}$ then also $G / N$ has maximal class.

(iii) If $N$ is a normal subgroup of $G$ of index $\geqslant p^{4}$ then $(G / N)_{1}=G_{1} / N$.

Lemma 3. Let $G$ be a p-group of maximal class of order $p^{n}$.

(i) $G$ has at most two different two-step centralisers: $G_{1}$ and $C_{G}\left(G_{n-2}\right)$. Moreover, $G_{1} \neq C_{G}\left(G_{n-2}\right)$ if and only if $l(G)=0$.

(ii) If $l(G) \geqslant 1$, every element in $G \backslash G_{1}$ is a uniform element.

THEOREM 3. Let $G$ be a 2-group of maximal class of order $2^{n}$ and let $M=\langle a\rangle$ be a cyclic maximal subgroup of $G$. Then:

(i) $G$ is isomorphic to one of the groups $D_{2^{n}}, S D_{2^{n}}$ or $Q_{2^{n}}$.

(ii) $M$ coincides with all the two-step centralisers $C_{G}\left(G_{i} / G_{i+2}\right)$. As a consequence, $G_{1}$ is cyclic and $G$ is a non-exceptional group.

Now, we prove the following new results for a $p$-group of maximal class.

Lemma 4. Let $G$ be a p-group of maximal class of order $p^{n}$ and let $M$ be a maximal subgroup of $G$ different from $G_{1}$ and from $C_{G}\left(G_{n-2}\right)$. Then there exist at 
least $n-3$ conjugacy classes of normaliser subgroups contained in $M$ and which are not contained in $G_{2}$.

ProOF: Let us observe (Lemma 3) that any $s \notin G_{1} \cup C_{G}\left(G_{n-2}\right)$ is a uniform element. Let us choose $s \in M \backslash\left(G_{1} \cup C_{G}\left(G_{n-2}\right)\right)$, which is clearly not in $G_{2}$. For any $1 \leqslant i \leqslant n-2$, let us define $H_{i}=\left\langle s, G_{i+1}\right\rangle$. Since by Theorem $1, s^{p} \in Z(G)=G_{n-1} \leqslant G_{i+1}$ for any $1 \leqslant i \leqslant n-2$, then $\left|H_{i}\right|=p^{n-i}$ for any $1 \leqslant i \leqslant n-2$, in particular $H_{1}=M$.

It is clear that, for any $2 \leqslant i \leqslant n-2, H_{i-1} \leqslant N_{G}\left(H_{i}\right)$. Conversely, since $G=\langle s\rangle G_{1}$, for any $x \in N_{G}\left(H_{i}\right)$ and $i$ fixed, then $x=s^{t} g$ for some $t$ and some $g \in G_{1}$ and, since $s \in N_{G}\left(H_{i}\right)$ it follows that $g \in N_{G}\left(H_{i}\right)$. On the other hand, for the mentioned $g \in G_{1}$, if $g \neq 1$ there exists some $j \in\{1, \ldots, n-1\}$ such that $g \in G_{j} \backslash G_{j+1}$ and then by Theorem $1,[s, g] \in G_{j+1} \backslash G_{j+2}$. If $j \geqslant i$ then clearly $g \in G_{i}, g \in H_{i-1}$ and, since also $s \in H_{i-1}$, we have $x \in H_{i-1}$, as desired. If not, $j \leqslant i-1$ that is, $j+1 \leqslant i$ and consequently $[s, g] \notin G_{i+1}$. But $g \in N_{G}\left(H_{i}\right)$ implies that $[s, g] \in H_{i} \cap G_{2}=G_{i+1}$, and we get a contradiction. In conclusion, since for any $2 \leqslant i \leqslant n-2$ the above $n-3$ normalisers, $H_{1}, \ldots, H_{n-3}$ have respectively the different orders $p^{n-1}, \ldots, p^{3}$, then they are non-conjugate normalisers and obviously they are not contained in $G_{2}$.

THEOREM 4 . Let $G$ be a p-group of maximal class of order $p^{n}$ and $G \supsetneqq Q_{2^{n}}$. Then $G$ has at least $p(n-3)+2$ conjugacy classes of normaliser subgroups.

Proof: Since $G$ is a $p$-group of maximal class, there are at least $p-1$ maximal subgroups of $G$, different from $G_{1}$ and from $C_{G}\left(G_{n-2}\right)$. Let us denote them as $M_{1}, M_{2}, \ldots, M_{p-1}$.

According to Lemma 4, for each $1 \leqslant l \leqslant p-1$ the corresponding $n-3$ normaliser subgroups in $M_{l}$ are not conjugate to each other because they have different orders, and also not conjugate to the ones of the same order found in a different maximal subgroup $M_{l^{\prime}}$ of $G$. Otherwise, since each $M_{l}$ is a normal subgroup then some uniform element would be contained in $M_{l} \cap M_{l^{\prime}}=\Phi(G)=G_{2}$, which is a contradiction. So, there are at least $(p-1)(n-3)$ non-conjugate classes of normalisers. Also, $G$ itself is another normaliser.

Now we distinguish two cases according to $G$ is or is not an exceptional group.

(i) Let $G$ be an exceptional group. In particular, $n \geqslant 6$. Now, the previously found $(p-1)(n-3)$ conjugacy classes of normalisers in Lemma 4 are, in particular, not contained in $C_{G}\left(G_{n-2}\right)$ and not in $G_{1}$. Furthermore, by Theorem 2 we know that $\bar{G}=G / Z(G)$, whose order is $p^{n-1}$, is a non-exceptional group. Now, since $G$ is an exceptional group, by Lemma 3 we get that $C_{G}\left(G_{n-2}\right) \neq G_{1}$ and, since $Z(G) \subseteq C_{G}\left(G_{n-2}\right)$ and $Z(G) \subseteq G_{1}$, we get that $\overline{C_{G}\left(G_{n-2}\right)} \neq \overline{G_{1}}=(\bar{G})_{1}$. On the other hand $\overline{C_{G}\left(G_{n-2}\right)}$ is a maximal subgroup of $\bar{G}$ so that, applying Lemma 4 for the non-exceptional $p$-group of maximal class $\bar{G}$, we get $n-4$ conjugacy classes of normaliser subgroups in $\bar{G}$ which are contained in $\overline{C_{G}\left(G_{n-2}\right)}$ and not in $(\bar{G})_{1}$. Obviously we get also $n-4$ conjugacy classes of normaliser subgroups in $G$ which are contained in $C_{G}\left(G_{n-2}\right)$ and not in $G_{1}$ and which are also clearly not 
conjugate to the previous ones. Hence, in this case, adding the normaliser subgroup $G$ there exist at least $(p-1)(n-3)+(n-4)+1=p(n-3)$ conjugacy classes of normaliser subgroups in $G$.

Let us take $g \in G_{1} \backslash G_{2}$ and $H=\left\langle g, G_{3}\right\rangle$. Since $G_{1} / G_{3} \cong C_{p} \times C_{p}$ and $g \notin G_{3}$ then $|H|=p^{n-2}$ and $H$ is a non-normal subgroup in $G$ because otherwise, the Lemma 2 implies that $H$ must be equal to $G_{2}$, which is a contradiction. Hence $\left|N_{G}(H)\right|$ must be equal to $p^{n-1}$. Since $H$ is a maximal subgroup of $G_{1}$, it is also a normal subgroup of it so that $G_{1}=N_{G}(H)$ : this is obviously a normaliser subgroup not conjugate to the previous ones.

Furthermore, in this case, by Theorem 2 , we know that $\exp G_{2}=p$. Now, let us take any $g_{0} \in G_{2} \backslash G_{3}$, so that $N_{G}\left(\left\langle g_{0}\right\rangle\right)=C_{G}\left(g_{0}\right)$ (Lemma 1). If we prove that $C_{G}\left(g_{0}\right)<G_{1}$, we have got at least another conjugacy class of normalisers not conjugate to the previous ones. To prove this, first of all we observe that any $x \in C_{G}\left(g_{0}\right)$ is contained in $G_{1}$. In fact, since $G_{2}=\left\langle g_{0}, G_{3}\right\rangle,\left[x, g_{0}\right]=1 \subseteq G_{4}$ and $\left[x, G_{3}\right] \subseteq G_{4}$, it is clear that $\left[x, G_{2}\right] \subseteq G_{4}$ and, in consequence, $x$ is an element of $G_{1}$. On the other hand, for some $g_{0} \in G_{2} \backslash G_{3}$, the centraliser $C_{G}\left(g_{0}\right)$ can be chosen different from $G_{1}$. If not, for any $g_{0} \in G_{2} \backslash G_{3}$ we have $C_{G}\left(g_{0}\right)=G_{1}$ so that we see that $G_{1}$ commutes with $\left\langle G_{2} \backslash G_{3}\right\rangle$. Furthermore, since $\left\langle G_{2} \backslash G_{3}\right\rangle=G_{2}$ we get that $G_{1}$ commutes with $G_{2}$ and in consequence, $G_{2} \subseteq Z\left(G_{1}\right)$. Now, since $G_{1} / G_{2}$ is a cyclic group, the quotient $G_{1} / Z\left(G_{1}\right)$ is also cyclic and in consequence $G_{1}$ is an Abelian group. Then $l(G)>0$ that is, $G$ is a non-exceptional group, which is a contradiction with the hypothesis.

(ii) Let $G$ be a non-exceptional group. In this case there are $p$ maximal subgroups different from $G_{1}=C_{G}\left(G_{n-2}\right)$ so that, according to Lemma 4, we get directly at least $p(n-3)$ conjugacy classes of normaliser subgroups not contained in $G_{1}$. Since $G$ itself is another normaliser, what we shall do next is to find another conjugacy class of normaliser subgroups not conjugate to the previous ones. To do this, we distinguish the cases $p>2$ and $p=2$.

(a) Let $p>2$. By Theorem 2 we know that $\exp G_{n-2}=p$. Let us take $g_{0}$ $\in G_{n-2} \backslash G_{n-1}$. Clearly, $G_{n-2}=\left\langle g_{0}, Z(G)\right\rangle$. Since $o\left(g_{0}\right)=p$, by Lemma 1 we have that $N_{G}\left(\left\langle g_{0}\right\rangle\right)=C_{G}\left(g_{0}\right)$. In this case $C_{G}\left(g_{0}\right)=C_{G}\left(G_{n-2}\right)=G_{1}$ so that $N_{G}\left(\left\langle g_{0}\right\rangle\right)=G_{1}$ and this normaliser in not conjugate to the previous ones.

(b) Let $p=2$. We know by Theorem 3 that $G_{1}$ is a cyclic group and in consequence, there exists only one element of order 2 in $G_{1}$, which is contained in $Z(G)$. Since $G \not Q_{2^{n}}$ there exists $g_{0} \in G \backslash G_{1}$ of order 2, which is in particular a uniform element. (Observe that by Theorem 3, we know that any 2-group of maximal class is a non-exceptional group and apply Lemma 3 ). In particular, for the uniform element $g_{0}$, applying the Theorem 1 we have that $\left|C_{G}\left(g_{0}\right)\right|=2^{2}$ and, since by Lemma 1 
$N_{G}\left(\left\langle g_{0}\right\rangle\right)=C_{G}\left(g_{0}\right)$, that $\left|N_{G}\left(\left\langle g_{0}\right\rangle\right)\right|=4$. On the other hand, since the $p(n-3)$ conjugacy classes of normalisers found previously are represented by subgroups whose order is greater than or equal to $2^{3}$, we have that $N_{G}\left(\left\langle g_{0}\right\rangle\right)$ represents another conjugacy class of normalisers not conjugate to the previous ones.

LEMMA 5. If $G \cong Q_{2^{n}}$ then $\omega(G)=2 n-5$.

Proof: Since any two conjugate non-normal subgroups in $G$ have conjugate normaliser subgroups and $G$ itself is the normaliser subgroup for all the normal subgroups in $G$, it is clear that $\omega(G) \leqslant \nu(G)+1$. Furthermore, if $G \cong Q_{2^{n}}$ we know that $\nu(G)=2 n-6$ (see [7]). Thus $\omega(G) \leqslant 2 n-5$.

On the other hand, for the non-exceptional 2-group $Q_{2^{n}}$, mimicking one part of the proof in Theorem 4 we get that $Q_{2^{n}}$ has at least $2(n-3)+1$ conjugacy classes of normaliser subgroups. It means that, $\omega(G) \geqslant 2 n-5$. In conclusion, $\omega(G)=2 n-5$.

NoTE. $G \cong Q_{2^{n}}$ is a counterexample for the bound in Theorem 4 .

Next, we study one case in which the inequality in Theorem 4 becomes an equality.

THEOREM 5 . Let $G$ be a $p$-group of maximal class of order $p^{n}$ and $G \not Q_{2^{n}}$. If $G_{1}$ is an Abelian group and for any $g \in G \backslash G_{1}, o(g)=p^{2}$ then $\omega(G)=p(n-3)+2$.

Proof: First of all we observe that in this case $G$ is a non-exceptional group. Let $H$ be any proper subgroup in $G$. We distinguish the cases $H \leqslant G_{1}$ and $H \not G_{1}$.

(a) For any $H$ subgroup in $G_{1}$ there are two possibilities for its normaliser. In fact, if $H \unlhd G$ then $N_{G}(H)=G$ and if not, since $H \subseteq G_{1}, G_{1}$ is Abelian and is a maximal subgroup in $G$, hence $G_{1}=N_{G}(H)$.

(b) For any $H \nsubseteq G_{1}$ there exists a maximal subgroup $M$ of $G$ with $M \neq G_{1}$, such that $H \leqslant M$. Looking at the structure of the subgroups in $G$, we get that there exists some $h \in M \backslash G_{2}, h \notin G_{1}$ and some $i \in\{1, \ldots, n-1\}$ such that $H=\left\langle h, G_{i+1}\right\rangle$. (Bear in mind that $h$ is a uniform element). Furthermore, since we know that $h^{p} \in Z(G)$ and $h \notin Z(G)$, we have that $\left\langle h, G_{n-1}\right\rangle$ has order $p^{2}$ and, since by hypothesis $o(h)=p^{2}$, we find that $\langle h\rangle=\left\langle h, G_{n}\right\rangle=\left\langle h, G_{n-1}\right\rangle$. Hence, for each fixed $h$ as above and each fixed $M$ maximal subgroup, arguing as in Lemma 4, we get $n-3$ non-conjugate proper normalisers:

$$
\left\langle h, G_{2}\right\rangle=N_{G}\left(\left\langle h, G_{3}\right\rangle\right), \ldots,\left\langle h, G_{n-2}\right\rangle=N_{G}\left(\left\langle h, G_{n-1}\right\rangle\right) .
$$

Next we shall prove that these conjugacy classes of normalisers do not depend on the choice of the element $h$. To do this, we shall prove that for any $k \in M \backslash G_{2}, k \notin G_{1}$ and $K=\left\langle k, G_{i+1}\right\rangle$ the normalisers $N_{G}(H)$ and $N_{G}(K)$ are conjugate in $G$. In fact, since $M=\langle h\rangle G_{2}=\langle k\rangle G_{2}$ and, by Theorem $1, k^{p} \in Z(G) \subseteq G_{2}$, then $h=k^{l} z$ for some $0<l \leqslant p-1$ and $z \in G_{2}$. Furthermore, since $(l, p)=1$, we see that $k^{l} \in M \backslash G_{1}$, that is, $k^{l}$ is also a uniform element (Lemma 3). By Theorem 1, we have that $\mathrm{Cl}_{G}\left(k^{l}\right)=k^{l} G_{2}$. 
Thus, $h \underset{G}{\sim} k^{l}$ and, since $G_{i} \unlhd G$, we find that

$$
\left\langle h, G_{i}\right\rangle=N_{G}(H) \underset{G}{\sim}\left\langle k^{l}, G_{i}\right\rangle=\left\langle k, G_{i}\right\rangle=N_{G}(K) .
$$

In conclusion, since there exist $p$ maximal subgroups in $G$ different from $G_{1}$, we get exactly $p(n-3)$ conjugacy classes of proper normalisers non-conjugate and not contained in $G_{1}$.

Furthermore, since we know that $G$ itself is another normaliser, that there is at most one conjugacy classes of normalisers contained in $G_{1}$ represented by $G_{1}$ and that, by Theorem 4 , there are at least $p(n-3)+2$ conjugacy classes of normalisers, we get the equality $\omega(G)=p(n-3)+2$.

\section{OTHER GENERAL RESULTS}

Definition 4: Let $G$ be a group. The norm of $G$, denoted by $\operatorname{Norm}(G)$, is the intersection of the normaliser subgroups of $G$ :

$$
\operatorname{Norm}(G)=\bigcap_{H \leqslant G} N_{G}(H)
$$

By a well known result $([8,6])$ the norm of a group $G$ contains the centre $Z(G)$ and is contained in the second term $Z_{2}(G)$ of the upper central series of $G$. The group $Z_{0}(G)$ is defined as the trivial subgroup of $G$.

In this section we want to prove the following theorem.

THEOREM 6 . If $G$ is a finite p-group ( $p$ odd) of nilpotency class $c$ then $\omega(G) \geqslant c$.

Before giving a proof, we need some preliminary results. From now on we shall use the letter $G$ to denote a finite $p$-group ( $p$ odd) whose nilpotency class is $c$.

LEMMA 6 . If $K$ is a normal subgroup of $G$ then $\omega(G) \geqslant \omega(G / K)$, moreover equality occurs if and only if for every subgroup $H \leqslant G$ there exists a subgroup $T \leqslant G$ containing $K$ such that $N_{G}(H)=N_{G}(T)$.

Proof: The preimages in $G$ of the normalisers in $G / K$ of the subgroups of $G / K$ are all the normalisers in $G$ of the subgroups of $G$ containing $K$. Since two subgroups of $G / K$ are conjugate in $G / K$ if and only if their preimages are conjugate in $G$ the inequality of the statement follows readily. If equality holds then every normaliser in $G$ is the preimage of a normaliser of $G / K$.

Corollary 1. Let $K$ be a normal subgroup of $G$. If $\omega(G)=\omega(G / K)$, then $K<Z_{2}(G)$ and $\operatorname{Norm}(G / K)=\operatorname{Norm}(G) / K$. Moreover if $Z(G) \leqslant K$ then we have that $\operatorname{Norm}(G)=Z_{2}(G)$ and $Z(G / K)=Z_{2}(G) / K=\operatorname{Norm}(G) / K$. 
Proof: By Lemma 6 it follows that for every subgroup $H \leqslant G$ there exists a subgroup $T_{H} \leqslant G$ containing $K$ such that $N_{G}(H)=N_{G}\left(T_{H}\right)$. If $K \leqslant H$ then we can choose $T_{H}=H$ so that

$$
\begin{aligned}
\operatorname{Norm}(G) K / K & =\left(\bigcap_{H \leqslant G} N_{G}(H)\right) K / K \\
& =\left(\bigcap_{H \leqslant G} N_{G}\left(T_{H}\right)\right) K / K \\
& =\bigcap_{K \leqslant T \leqslant G} N_{G}(T) / K \\
& =\operatorname{Norm}(G / K) .
\end{aligned}
$$

Hence $K \leqslant \operatorname{Norm}(G) \leqslant Z_{2}(G)$.

Note that $\operatorname{Norm}(G) \leqslant Z_{2}(G)$, therefore if $Z(G) \leqslant K$ then

$$
Z_{2}(G) / K \leqslant Z(G / K) \leqslant \operatorname{Norm}(G / K)=\operatorname{Norm}(G) / K \leqslant Z_{2}(G) / K
$$

so that every inequality in the previous chain is actually an equality. This implies that $\operatorname{Norm}(G)=Z_{2}(G)$.

We remind the reader that a group $T$ is capable if there exists a group $H$ such that $H / Z(H) \cong T$. The following result appears as part of Theorem 3 and Theorem 4 in [1].

Lemma 7. Let $G$ be a finite p-group. One of the following holds true:

(i) $\operatorname{Norm}(G) / Z(G)$ is cyclic,

(ii) $[G, \operatorname{Norm}(G)]$ is cyclic.

Moreover if $[G, \operatorname{Norm}(G)]$ is cyclic and non-trivial then $G$ is not capable.

Definition 5: Let $G$ be a finite p-group of nilpotency class $c$ and $N$ a normaliser subgroup of $G$. We shall say that $N$ is of level $i$ if $i=\sup \left\{h \mid Z_{h}(G) \leqslant N\right\}$.

REMARK 1. Note that two normaliser subgroups of $G$ having different levels cannot be conjugate. We shall use this fact in the following without any further reference.

Lemma 8. Suppose that $c \geqslant 2$. If $\operatorname{Norm}(G) \neq Z_{2}(G)$ then there exists a normaliser subgroup $N$ of $G$ of level 1 .

Proof: By hypothesis there exists an element $x \in Z_{2}(G)$ and a subgroup $H \leqslant G$ such that $x \notin N_{G}(H)$ : let $N=N_{G}(H)$.

Trivial consequences are the following Corollaries.

Corollary 2. If $G$ is Abelian or Norm $\left(G / Z_{i}(G)\right) \neq Z_{2}\left(G / Z_{i}(G)\right)$ for every $i$ in the set $\{0, \ldots, c-2\}$, then for every $j$ in the set $\{1, \ldots, c\}$ there exists at least one normaliser subgroup $N_{j}$ of level $j$. In particular $\omega(G) \geqslant c$.

Proof: Taking quotients with respect to the terms of the upper central series and using inductively Lemma 8 we are reduced to prove that an Abelian group $G$ has a normaliser $N$ of level 1 : let $N=G$ in this case. 
CoRollary 3. If $G$ is a finite p-group of nilpotency class $c \leqslant 2$ then $\omega(G) \geqslant c$.

Proof: If $G$ is Abelian then $\omega(G)=1=c$. Suppose now that $G$ has nilpotency class $c=2$. Note that $G=Z_{2}(G)$ cannot be equal to $\operatorname{Norm}(G)$, since otherwise $G$ would be a Dedekind $p$-group with $p$ odd, that is, an Abelian group. Thus Corollary 2 yields the claim for this case.

Lemma 9. Suppose that $G$ is non-Abelian and that there exists $d \in Z_{2}(G)$ such that $H=[d, G]$ is cyclic of order $p$. If the set of the elements of $G$ having a non-trivial power in $H$ is contained in a maximal subgroup $T$ of $G$ then $d \in Z_{2}(G) \backslash \operatorname{Norm}(G)$ and $G$ has at least two non-conjugate normaliser subgroups of level 1 not containing $d$.

Proof: Let $C=C_{G}(d)$. The subgroup $C$ is a maximal subgroup of $G$ being the kernel of the non-trivial homomorphism defined on $G$ by $g \rightarrow[d, g] \in H$. Choose an element $x \in G \backslash(C \cup T)$, since $[x, d] \neq 1$ and $x$ has no non-trivial power in $H$, it follows that $d \notin N_{G}(\langle x\rangle)$ and $d \notin \operatorname{Norm}(G)$. Let $V$ be a maximal subgroup of $G$ containing $N=N_{G}(\langle x\rangle)$, since $x \notin C$ we have that $V \neq C$. Thus $K=C \cap V$ is a normal subgroup of $G$ of index $p^{2}$. Choose an element $y \in C \backslash K$ and for every $i$ in the set $\{1, \ldots, p\}$ let $x_{i}=x y^{i-1}$. Assume by way of contradiction that $x_{i}, x_{j} \in T$ where $i \neq j$. It follows that $y^{i-j}=x_{j}^{-1} x_{i} \in T$, so that $y \in T$ and also $x \in T$ : a contradiction. Thus there exists at least one index $i_{0}>1$ such that $x_{i_{0}} \notin T$ and, as a consequence, if we let $M=N_{G}\left(\left\langle x_{i_{0}}\right\rangle\right)$, we see that $d \notin M$. We have shown that the two normaliser subgroups $M$ and $N$ are of level 1.

Now we show that $N$ and $M$ are not conjugate. We shall use the bar notation to denote the projections on $G / K$ of elements and subgroups of $G$. Assume by way of contradiction that $\bar{x}_{i_{0}} \in \bar{N}$, then $\bar{y}^{1-i_{0}}=\bar{x}_{i_{0}}^{1} \bar{x} \in \bar{N}$ and also $\bar{y} \in \bar{N}$. This yields $\bar{N}=\bar{G}$. Therefore $|V / K| \geqslant|N K / K|=|\bar{N}|=|\bar{G}|=p^{2}$, a contradiction since $K$ is a maximal subgroup of $V$. It follows that $\bar{M} \neq \bar{N}$ so that $\bar{M}$ and $\bar{N}$ cannot be conjugate subgroups in the Abelian group $G / K$. As a consequence $M$ and $N$ cannot be conjugate subgroups of $G$.

Lemma 10. If $G$ is capable non-Abelian and $[d, G]$ is cyclic for some element $d \in Z_{2}(G) \backslash Z(G)$ then $G$ has at least two non-conjugate normaliser subgroups of level 1 .

Proof: We can assume that $d$ has order $p$ modulo $Z(G)$. Let $S$ be the set of elements of $G$ having a non-trivial power in the cyclic group $H=[d, G]$. The set $S$ cannot generate $G$, otherwise $G$ would be not capable (since the preimage of the cyclic subgroup $H$ would be central in every central extension of $G$ ). It follows that $S$ is contained in a maximal subgroup $T$ of $G$. The claim now follows readily from Lemma 9 .

A straightforward consequence is the following corollary.

CoRollary 4. If $G$ is capable non-Abelian and $Z(G)$ is cyclic then $G$ has at least two non-conjugate normaliser subgroups of level 1. 
Lemma 11. If $A$ is an $A$ belian finite group, $B$ a non trivial subgroup of $A$ and $M$ a subgroup of $A$ maximal with respect to the property of being a subgroup of $A$ not containing $B$, then $A / M$ is cyclic of prime power order.

PROof: Every non-trivial subgroup of $A / M$ contains $B M / M$, since otherwise its preimage in $A$ would not contain $B$ and would contain properly $M$, in spite of the maximality of $M$. Thus the finite Abelian group $A / M$, which is the direct product of cyclic groups of prime power order, has a unique minimal subgroup and therefore $A / M$ itself is necessarily cyclic of prime power order.

LEMMA 12. Suppose that $G$ has nilpotency class $c \geqslant 2$. If

$$
\operatorname{Norm}(G / K)=Z(G) / K=\operatorname{Norm}(G) / K
$$

for every subgroup $K$ of $Z(G)$ not containing $\gamma_{c}(G)$ then one of the following occurs:

(i) $Z_{2}(G) / Z(G)$ is cyclic, $c \geqslant 3$ and $G$ has one normaliser subgroup of level 1 and two non-conjugate normaliser subgroups of level 2,

(ii) $Z_{2}(G) / Z(G)$ is not cyclic and $G$ has two non conjugate normaliser subgroup of level 1 .

Proof: Assume first that $Z_{2}(G) / Z(G)$ is cyclic. Since $G / Z(G)$ cannot be nontrivial cyclic, the group $G$ has nilpotency class $c \geqslant 3$. Also Norm $(G)=Z(G) \neq Z_{2}(G)$ so that, by Lemma 8 , the group $G$ has a normaliser subgroup of level 1. Applying Corollary 4 to the capable group $G / Z(G)$ we find that $G$ has also two non conjugate normaliser subgroups of level 2.

Now we suppose that $Z_{2}(G) / Z(G)$ is not cyclic. Let $d_{1}$ and $d_{2}$ be two elements in $Z_{2}(G)$ of order $p$ modulo $Z(G)$ such that $\left\langle d_{1}\right\rangle Z(G) \neq\left\langle d_{2}\right\rangle Z(G)$. Let $H$ be a subgroup of $Z(G)$ maximal in the set $\left\{K \leqslant Z(G) \mid \gamma_{c}(G) \$ K\right\}$. We shall use the bar notation to denote the projections of elements and subgroups of $G$ over $\bar{G}=G / H$. By our hypotheses we have $\operatorname{Norm}(\bar{G})=Z(G) / H=Z(\bar{G})$. Also, by Lemma 11, we find that $Z(\bar{G})=Z(G) / H$ is a cyclic group. Thus there exists $\bar{g}_{1} \in \bar{G}$ that is not normalised by $\bar{d}_{1}$ and two integers $\lambda_{1}$ and $\lambda_{2}$ that are nor both divisible by $p$ such that $\bar{d}_{3}=\bar{d}_{1}^{\lambda_{1}} \bar{d}_{2}^{\lambda_{2}}$ centralises $\bar{g}_{1}$. Clearly $N_{G}\left(\left\langle g_{1}\right\rangle H\right)$ contains the normal subgroup $\left\langle d_{3}\right\rangle Z(G)$ and is of level 1 since it does not contains $d_{1}$. Using the same argument with $d_{3}$ in place of $d_{1}$ we find an element $g_{2} \in G$ such that $N_{G}\left(\left\langle g_{2}\right\rangle H\right)$ is of level 1 and does not contain $d_{3}$. In particular it does not contain the normal subgroup $\left\langle d_{3}\right\rangle Z(G)$ so that it cannot be conjugate to $N_{G}\left(\left\langle g_{1}\right\rangle H\right)$.

LEMMA 13. Suppose that $G$ is non-Abelian and capable. One of the following occurs:

(i) $\operatorname{Norm}(G) \neq Z_{2}(G)$ and $G$ has a normaliser subgroup of level 1,

(ii) $\operatorname{Norm}(G)=Z_{2}(G)$ and $G$ has two non-conjugate normaliser subgroups of level 2 and $c \geqslant 3$. 
Proof: By Corollary 3 we can assume $c \geqslant 3$ and by Lemma 8 that $\operatorname{Norm}(G)$ $=Z_{2}(G)$. Lemma 7 implies that $Z_{2}(G) / Z(G)=\operatorname{Norm}(G) / Z(G)$ is cyclic and is the centre of the capable group $\bar{G}=G / Z(G)$. By Corollary 4 the group $\bar{G}$ has two nonconjugate normaliser subgroups of level 1 . Their preimages in $G$ are two normaliser subgroups of level 2 in $G$ that are not conjugate.

THEOREM 7. If $G$ is a capable finite p-group ( $p$ odd) of nilpotency class $c$ then $\omega(G) \geqslant c$.

Proof: The statement is trivial if $G$ is Abelian. Otherwise, by Lemma 13, we have that $\omega(G) \geqslant 1+\omega(G / Z(G))$ if $G$ has a normaliser subgroup of level 1 or $\omega(G)$ $\geqslant 2+\omega\left(G / Z_{2}(G)\right)$ if $c \geqslant 3$ and $G$ has two non-conjugate normaliser subgroups of level 2. The claim now follows by arguing induction on the nilpotency class of $G$.

Now we are in a position to prove the main result in this section.

ProOF OF THEOREM 6: We shall use the bar notation to denote the projection of elements and subgroups of $G$ onto $\bar{G}=G / Z(G)$. By Corollary 3 we can assume $c \geqslant 3$.

If $\operatorname{Norm}(G) \neq Z_{2}(G)$ then Lemma 8 (or Corollary 1 ) yields $\omega(G) \geqslant 1+\omega(\bar{G})$. Since $\bar{G}$ is capable, Theorem 7 shows that $\omega(\bar{G}) \geqslant c-1$ so that $\omega(G) \geqslant c$ as required.

Suppose now that $\operatorname{Norm}(G)=Z_{2}(G)$. By Theorem 7, we can assume that $G$ is not capable. We have $\omega(G) \geqslant \omega(\bar{G}) \geqslant c-1$ since $\bar{G}$ is a capable group of nilpotency class $c-1$.

Assume, by way of contradiction, that $\omega(G)<c$ and that $c$ is minimal with respect to this property. We have $c \geqslant 3$ and $\omega(G)=\omega(\bar{G})=c-1$, hence, by Corollary 1 , we see that $\operatorname{Norm}(\bar{G})=Z(\bar{G})$. If $\bar{K}$ is a subgroup of $Z(\bar{G})$ not containing $\gamma_{c-1}(\bar{G})$ then $\bar{G} / \bar{K}$ has nilpotency class $c-1$, so that $c-1 \leqslant \omega(\bar{G} / \bar{K}) \leqslant \omega(\bar{G})=c-1$. Applying once more Corollary 1 we have that $\operatorname{Norm}(\bar{G}) / \bar{K}=\operatorname{Norm}(\bar{G} / \bar{K})=Z(\bar{G}) / \bar{K}$. We have shown that $\bar{G}$ satisfies the hypotheses of Lemma 12 so that, arguing induction, either $w(G)=\omega(\bar{G})$ $\geqslant 2+\omega(\bar{G} / Z(\bar{G})) \geqslant c$ or $G$ has class $c \geqslant 4$ and $w(G)=\omega(\bar{G}) \geqslant 3+\omega\left(\bar{G} / Z_{2}(\bar{G})\right) \geqslant c$. In both cases we have a contradiction.

\section{REFERENCES}

[1] J.C. Beidleman, H. Heineken and M. Newell, 'Centre and norm', Bull. Austral. Math. Soc. 69 (2004), 457-464.

[2] N. Blackburn, 'On a special class of p-groups', Acta Math. 100 (1958), 45-92.

[3] G.A. Fernández-Alcober, 'An introduction to finite p-groups: regular p-groups and groups of maximal class', Mat. Contemp. 20 (2001), 155-226.

[4] G.A. Fernández-Alcober and L. Legarreta, 'On the number of conjugacy classes of non-normal subgroups in a finite $p$-group', (preprint).

[5] The GAP Group, 'GAP - Groups, algorithms, and programming, version 4.4', (2004) (http://www.gap-system.org).

[6] W. Kappe, 'Die a-norm einer gruppe', Mlinois J. Math. 5 (1961), 187-197. 
[7] J. Poland and A. Rhemtulla, 'The number of conjugacy classes of non-normal subgroups in nilpotent groups', Comm. Algebra 24 (1996), 3237-3245.

[8] E. Schenkman, 'On the norm of a group', Ilinois J. Math. 4 (1960), 150-152.

Dipartimento di Matematica Pura ed Applicata

Università di L'Aquila

Via Vetoio, 67010 Coppito (L'Aquila)

Italy

e-mail: gavioli@univaq.it

Dipartimento di Matematica e Informatica Università di Salerno

Via Ponte don Melillo

84084 Fisciano (SA)

Italy

e-mail: csica@unisa.it
Matematika Saila

Euskal Herriko Unibertsitatea

48080 Bilbo

Spain

e-mail: mtplesol@lg.ehu.es

Dipartimento di Matematica e Informatica Università di Salerno

Via Ponte don Melillo

84084 Fisciano (SA)

Italy

e-mail: mtota@unisa.it 